\title{
Pregabalin-induced hyperprolactinemia in a patient with fibromyalgia: A case report
}

\author{
Aslihan Taraktas, ${ }^{1}$ Nilgun Mesci, ${ }^{2}$ Gulcan Ozturk, ${ }^{3}$ Duygu Geler Kulcu, ${ }^{2}$ Ece Aydog ${ }^{4}$ \\ ${ }^{1}$ Department of Physical Medicine and Rehabilitation, Fatih Sultan Mehmet Training and Research Hospital, Istanbul, Turkey \\ 2Department of Physical Medicine and Rehabilitation, Haydarpasa Numune Training and Research Hospital, Istanbul, Turkey \\ ${ }^{3}$ Department of Physical Medicine and Rehabilitation, Gebze State Hospital, Kocaeli, Turkey \\ ${ }^{4}$ Department of Physical Medicine and Rehabilitation, Yeditepe University Hospital, Istanbul, Turkey
}

\begin{abstract}
Several pharmacological and non-pharmacological modalities have been proposed for the treatment of fibromyalgia syndrome (FMS), a common rheumatic disease. Pregabalin is suggested as a first-step medication for FMS in the newest guidelines. Drowsiness, dizziness, and peripheral edema are well-known side effects of pregabalin; however, mastalgia is rarely seen. Presently described is a case of FMS in a patient who developed mastalgia and hyperprolactinemia (HPL) while taking pregabalin.
\end{abstract}

Keywords: Fibromyalgia; hyperprolactinemia; neuropathic pain; pregabalin.

Cibromyalgia syndrome (FMS) causes wide- spread pain and is often accompanied by fatigue, tenderness, memory problems and sleep disturbances. It affects $2-4 \%$ of the population. According to the American College of Rheumatology (ACR) FMS 1990 criteria, widespread pain lasting at least 3 months, and 11 of 18 positive tender points are necessary for the diagnosis [1]. In 2011, the ACR recommended new modified diagnostic criteria for FMS (2011 ModCr). New criteria consist of 19 objective pain locations in addition to 6 self-reported symptoms (impaired sleep, fatigue, poor cognition, headaches, depression and abdominal pain) [2]. Diagnosis is based on widespread pain index (WPI) and symptom severity (SS) scores of 3-6 WPI and $\geq 9$ SS or $\geq 7$ WPI and $\geq 5$ SS.

The etiopathophysiology of FMS is still unknown; however, neuroendocrine, autonomic, immunological mechanisms, genetic and environmental factors have been suspected. Recent research has supported the role of central sensitization mechanism and involves new treatment options [3]. Pregabalin, one of the first-step drugs for the treatment of neuropathic pain, has also been used for the treatment of FMS in recent years $[4,5]$. This case report discusses a patient with FMS who developed mastalgia and hyperprolactinemia (HPL), believed to be side effects of pregabalin. 


\section{CASE REPORT}

A 32-year-old female patient with symptoms of widespread pain, fatigue and sleep problems presented at the outpatient clinic one year ago. She did not have a history of chronic disease or any medication use. Physical examination indicated that range of motion of spine and peripheric joints were not limited and painful. There were no pathological findings in the neurological examination. She had 11 positive tender points (according to ACR 1990 FMS classification criteria) and her WPI and SS scores were 9 and 5 , respectively.

Routine laboratory tests (hemoglobin [Hgb]: $13.3 \mathrm{~g} / \mathrm{dL}$, aspartate aminotransferase [AST]: 22 $\mathrm{U} / \mathrm{L}$, alanine aminotransferase [ALT]: $18 \mathrm{U} / \mathrm{L}$, creatinine: $0.49 \mathrm{mg} / \mathrm{dL}$, thyroid stimulating hormone [TSH]: $0.707 \mathrm{uIU} / \mathrm{mL}$, sedimentation: 14 $\mathrm{mm} / \mathrm{h}$ and C-reactive protein: $5 \mathrm{mg} / \mathrm{L}$ ) were in normal ranges. With these findings, she was diagnosed with FMS according to 2011 ModCr and ACR 1990 criteria.

Pregabalin was prescribed with a dosage of 75 $\mathrm{mg} /$ day to be taken at night for the first three days. The dosage was then increased to $75 \mathrm{mg}$ twice a day for 1 week. During this period, the patient complained of drowsiness and dizziness; however, the effects were not severe enough to require discontinuing the medication. In the second week, the pregabalin dose was $150 \mathrm{mg}$ twice a day. Patient began to tolerate the drug well. During treatment period of 1 year, follow-up examinations were conducted at 3 -month intervals. Over the course of the year, intensity of symptoms decreased. Patient's visual analog scale pain score and fibromyalgia impact questionnaire score decreased from 8 to 4 and from 50 to 33 , respectively, over time. Patient was also asked about drug side effects, such as vertigo, drowsiness, dry mouth, and weight gain.

In her last examination, one year after the prescription, the patient complained of mastalgia and tenderness in her breasts without galactoria. Gynecology and endocrinology departments were consulted.

Laboratory test results were as follows: serum prolactin (PRL): $54.2 \mathrm{ng} / \mathrm{mL}(3.2-26)$, TSH: $1.45 \mathrm{IU} / \mathrm{mL}$, serum B12 vitamin: $223 \mathrm{pg} / \mathrm{mL}$, erythrocyte sedimentation rate: $22 \mathrm{mmHg} / \mathrm{h}$, serum dehydroepiandrosterone sulphate (DHEA-S): 556 $\mathrm{mg} / \mathrm{dL}$, serum follicle stimulating hormone (FSH): 7.44, serum luteinizing hormone (LH): 11.35 IU/L, serum estradiol: $37 \mathrm{pg} / \mathrm{mL}$, fasting blood sugar: $100 \mathrm{mg} / \mathrm{dL}$, and serum insulin: $4.8 \mathrm{IU} / \mathrm{mL}$. Breast ultrasound and cranial magnetic resonance imaging (MRI) showed no abnormality.

HPL etiology was not observed; therefore HPL was considered a side effect of pregabalin. Accordingly, use of pregabalin was ceased. It was explained to the patient that after stopping the medication, the complaints might resolve themselves over time. However, the patient did not want to wait for her complaints to resolve in time, and so kebergolin $0.5 \mathrm{mg} /$ day for one week was prescribed. After one week of use, PRL value decreased to $2.44 \mathrm{ng} / \mathrm{mL}$ and breast tenderness and mastalgia complaints were completely resolved. As patient did not want to use any further medication, an exercise and physical therapy program was scheduled.

\section{DISCUSSION}

FMS is a multifactorial disease of unknown etiology with several contributing factors. These include hormones, immune system, external stressors, psychiatric aspects, and dysfunction of central and autonomic nervous systems [5]. Recent studies suggest that FMS is a neurosensitive disorder characterized by abnormal processing of pain. However, etiopathogenesis is related to central nerve system changes and central sensitization. Various neurotransmitters (i.e., serotonin, norepinephrine, dopamine, substance $\mathrm{P}$ ) seem to be involved in central sensitization [5].

There are various pharmacological treatment recommendations for FSM. European League Against Rheumatism (EULAR) primarily advises the use of pregabalin, duloxetine, and amitriptyline. Guidelines suggest pregabalin in first-line treatment $[4,5]$.

In cases of FMS, voltage-sensitive calcium channels remain open longer, an effect of an increase in 
the number of alpha 2-delta subunits. This causes greater glutamate release in presynaptic gap and increase in superficial dorsal root neurons excitability. Therefore, pregabalin can be used to treat FMS.

Pregabalin is a medication that is structurally analogous to gamma-amino butyric acid (GABA). It is usually prescribed for chronic pain syndromes such as postherpetic neuralgia, diabetic and peripheric neuropathic pain, partial seizures, sleep disorders, anxiety disorders, and fibromyalgia [6].

Pregabalin, an alpha 2 delta Ca channel ligand, has analgesic, anxiolytic and anticonvulsive properties. Being a calcium channel antagonist, it decreases the release of many neurotransmitters such as substance $\mathrm{P}$, norepinephrine, and glutamate [6-8].

Common adverse effects of pregabalin include dizziness, vertigo, nausea, vomiting, hypotension, headache, hallucinations, fatigue, cognitive impairment, drowsiness, and peripheric edema [9]. Constipation, rhabdomyolysis, hypersensitivity reaction, akathisia, urticaria, and mastalgia are rarely encountered adverse effects and provided as prospectus information. In the present case, HPL, identified as a rare premarketing side effect that has not been reported in the literature, was observed [10].

HPL is a common disorder of pituitary gland that may occur for various reasons. The most frequent cause is PRL-secreting tumors. Before examining for pituitary tumors as part of differential diagnosis, pregnancy, primary hypothyroidism, liver or renal failure, polycystic ovary syndrome, and medication should be considered. In the present case, gynecology and endocrinology departments were consulted in order to analyze those etiologic factors. According to results of physical examinations, laboratory tests and MRI findings, the patient's HPL was thought to be drug-induced. Medications are the most frequent cause of non-tumoral HPL. In the case of drug-induced HPL, PRL levels range from 25 to $100 \mathrm{ng} / \mathrm{mL}$, similar to the present patient's PRL level $[11,12]$.

The results of laboratory and diagnostic tests were in normal range. Thus, HPL may have been induced by medication. In the literature, several drugs are reported to cause HPL, such as antipsychotics, antidepressants (e.g., tricyclic and tetracyclic selective serotonin reuptake inhibitors [SSRIs]), opiates, antihypertensive medications (verapamil, methyldopa, reserpine), gastrointestinal medications (metoclopramide, domperidone, histamine receptor blockers) and estrogens [13].

Dopamine (prolactin inhibiting factor [PIF]) is the most important hypothalamic-inhibiting factor of PRL. It binds to the D2 receptor, affecting lactotroph (unique among endocrine cells in having a high basal secretory activity, and which are located in the anterior pituitary gland) cell membranes. This activation causes suppression of the PRL gene expression and inhibition of PRL exocytosis; first, inhibition of adenylyl cyclase and inositol phosphate metabolism, then modification/alteration of several potassium and calcium channels [14].

Antipsychotics like phenothiazines (chlorpromazine-fluphenazine), thioxanthenes (thiothixene), and butyrophenones (haloperidol) block dopamine and can lead to development of HPL. In addition, gastrointestinal medications used to increase gastrointestinal motility may cause HPL in the same way, by blocking dopamine receptors. Most evidence suggests that opioid peptides do not directly affect the pituitary gland and stimulate PRL release by inhibiting hypothalamic dopamine secretion [13]. Among antihypertensive medications, only verapamil raises prolactin levels. Kelley et al., researched the mechanism of this effect of verapamil, a calcium channel blocker. Researchers concluded that verapamil decreases central dopamine generation, perhaps through N-type calcium channels [15].

Several peptides or neurotransmitters, such as serotonin, oestrogens, tachykinins (substance $\mathrm{P}$, neurokinin A-B and neuropeptide K), Gonadotropin-releasing hormone associated peptide (GAP), opioids (especially $ß$-endorphins), histamine and GABA can also affect the modulation of PRL secretion [11].

GABA, a neurotransmitter secreted mostly in the hypothalamus, has a mostly inhibiting effect. GABA modulates secretion of pituitary hormones through dopaminergic tone or influence on the an- 
terior pituitary cells. Stimulation of GABA-A and GABA-B receptor decreases PRL secretion; however, Nakayama et al., reported that GABA-C receptor activation led to an increase in PRL levels in cultured rat anterior pituitary cells [16].

Pregabalin is an analog of GABA, but it shows no effect on GABA receptors. Activation mechanism is disclosed with calcium channel blocking. Though it cannot be precisely explained how pregabalin induced HPL in the present case, only the use of pregabalin accounts for its occurrence.

In conclusion, this is the first case report demonstrating HPL due to pregabalin usage. Although it has been identified as a premarketing adverse side effect, there is no recent case report in the literature. As it is a very rare side effect, in clinical practice, mastalgia is generally not questioned, though the most frequent side effects are routinely questioned. Based on this case report, when pregabalin is used, the authors suggest professionals also inquire about breast tenderness. Further studies should focus on the effects of pregabalin use on prolactin levels.

\section{Conflict of Interest: None declared.}

Financial Disclosure: The authors declared that this study has received no financial support.

Authorship contributions: Concept - A.T., N.M., D.G.K.; Design - A.T., N.M., D.G.K.; Supervision - D.G.K; Data collection and/or processing - A.T., G.O.; Analysis and/or interpretation - A.T., N.M., G.O., D.G.K, E.A; Literature search - A.T.; Writing - A.T., D.G.K; Critical review - D.G.K., E.A.

\section{REFERENCES}

1. Wolfe F, Smythe HA, Yunus MB, Bennett RM, Bombardier C, Goldenberg DL, et al. The American College of Rheumatology 1990 Criteria for the Classification of Fibromyalgia. Report of the Multicenter Criteria Committee. Arthritis Rheum 1990;33:160-72. Crossret

2. Wolfe F, Clauw DJ, Fitzcharles MA, Goldenberg DL, Häuser W, Katz RS, et al. Fibromyalgia criteria and severity scales for clinical and epidemiological studies: a modification of the ACR Preliminary Diagnostic Criteria for Fibromyalgia. J Rheumatol
2011;38:1113-22. Crossret

3. Yunus MB. Central sensitivity syndromes: a new paradigm and group nosology for fibromyalgia and overlapping conditions, and the related issue of disease versus illness. Semin Arthritis Rheum 2008;37:339-52. Crossre

4. Ablin J, Fitzcharles MA, Buskila D, Shir Y, Sommer C, Häuser W. Treatment of fibromyalgia syndrome: recommendations of recent evidence-based interdisciplinary guidelines with special emphasis on complementary and alternative therapies. Evid Based Complement Alternat Med 2013;2013:485272

5. Bellato E, Marini E, Castoldi F, Barbasetti N, Mattei L, Bonasia DE, et al. Fibromyalgia syndrome: etiology, pathogenesis, diagnosis, and treatment. Pain Res Treat 2012;2012:426130. Crossre

6. Bockbrader HN, Wesche D, Miller R, Chapel S, Janiczek N, Burger P. A comparison of the pharmacokinetics and pharmacodynamics of pregabalin and gabapentin. Clin Pharmacokinet 2010;49:661-9. Crossrel

7. Gee NS, Brown JP, Dissanayake VU, Offord J, Thurlow R, Woodruff GN. The novel anticonvulsant drug, gabapentin (Neurontin), binds to the alpha2delta subunit of a calcium channel. J Biol Chem 1996;271:5768-76. Crossre]

8. Fink K, Dooley DJ, Meder WP, Suman-Chauhan N, Duffy S, Clusmann $\mathrm{H}$, et al. Inhibition of neuronal $\mathrm{Ca}(2+)$ influx by gabapentin and pregabalin in the human neocortex. Neuropharmacology 2002;42:229-36. Crossre

9. Salinsky M, Storzbach D, Munoz S. Cognitive effects of pregabalin in healthy volunteers: a double-blind, placebo-controlled trial. Neurology 2010;74:755-61. Crossre

10. GD-pregabalin (pregabalin) Product Monograph. Pfizer Canada Inc. 2013:1-63.

11. Kabalak T, Yilmaz C, Tuzun M. Endokrinoloji. El Kitabı, Izmir Güven Kitabevi; 2004. s. 61-92.

12. Erem C, Civan N, Nuhoğlu İ. Causes and Differential Diagnosis of Hyperprolactinemia. Turkiye Klinikleri J Endocrin-Special Topics 2012;5:10-21.

13. Molitch ME. Medication-induced hyperprolactinemia. Mayo Clin Proc 2005;80:1050-7. Crossre

14. López Román J, Martínez Gonzálvez AB, Luque A, Pons Miñano JA, Vargas Acosta A, Iglesias JR, et al. The effect of a fibre enriched dietary milk product in chronic primary idiopatic constipation. [Article in Spanish] Nutr Hosp 2008;23:12-9. [Abstract]

15. Kelley SR, Kamal TJ, Molitch ME. Mechanism of verapamil calcium channel blockade-induced hyperprolactinemia. Am J Physiol 1996;270(1 Pt 1):96-100.

16. Nakayama Y, Hattori N, Otani H, Inagaki C. Gamma-aminobutyric acid (GABA)-C receptor stimulation increases prolactin (PRL) secretion in cultured rat anterior pituitary cells. Biochem Pharmacol 2006;71:1705-10. Crosste. 